\title{
The Characterization of Linear Polyethylene SRM 1475. II. Determination of Total Methyl Content by Infrared Spectrophotometry.
}

\author{
James E. Brown \\ Institute for Materials Research, National Bureau of Standards, Washington, D.C. 20234
}

(December 15, 1971)

\begin{abstract}
An ASTM method was used to determine the methyl content of linear polyethylene SRM 1475 by measuring the absorbance at $1378 \mathrm{~cm}^{-1}(7.25 \mathrm{~nm})$ of methyl groups on compensated infrared spectra. The methyl content of this polymer was found to be 0.15 methyls per 100 carbon atoms. Considering the methyls to be polymer end groups, the number average molecular weight computed approximates that determined by gel permeation chromatography within the experimental error. These values indicate that the polymer is essentially free of branching.
\end{abstract}

Key words: Infrared; linear polyethylene; methyl; methylene; number average molecular weight; spectra; spectrophotometry.

\section{Introduction}

The total methyl group content in polyethylene may be used to estimate the amount of branching in the polymer. If, for example, it is assumed that all chainends are methyl groups, then the methyl-group content of the polymer may be combined with its numberaverage molecular weight, $M_{n}$, to yield an estimate of the average number of chain ends per molecule.

In the characterization of SRM 1475, the total methyl content was determined to obtain an estimate of the polymer's linearity. Infrared spectrophotometry was used to determine the methyl content from the absorbance at about $1378 \mathrm{~cm}^{-1}$ due to methyl groups. This band is overlapped by bands at 1368 and $1352 \mathrm{~cm}^{-1}$, and therefore cannot be used directly to estimate methyl group content. However, it has been shown $[1-5]^{1}$ that both the latter two bands and another band at $1304 \mathrm{~cm}^{-1}$ arise from methylene-groups in the amorphous or disordered regions only, while the 1378 $\mathrm{cm}^{-1}$ band arises from methyl groups in both amorphous and crystalline regions. If we assume [5] that the spectrum of solid polyethylene is a superposition of contributions from amorphous and crystalline regions, then the spectrum arising from methyl groups alone may be obtained as a difference spectrum between two films of the same polymer, but different degrees of crystallinity and appropriately different thicknesses.

In this paper, we report the results for total methyl content in SRM 1475 by the method described in American Society for Testing and Materials (ASTM) Designation: D 2238-68, "Standard Method of Test for Absorbance of Polyethylene Due to Methyl Groups

\footnotetext{
${ }^{1}$ Figures in brackets indicate the literature references at the end of this paper.
}

at $1378 \mathrm{~cm}^{-1} .{ }^{2}$ By this method, the absorbance is measured at the methyl frequency $\left(1378 \mathrm{~cm}^{-1}\right)$ on films which are largely crystalline while compensating for the amorphous methylene absorbance with thinner, less crystalline films. Graphically, the absorbance resulting only from methyl groups is interpolated to the point of zero methylene absorbance at $1304 \mathrm{~cm}^{-1}$. Each of the absorbances at 1378 and $1304 \mathrm{~cm}^{-1}$ is corrected for the effect produced by the difference in thickness and density of the crystalline and amorphous films. The resulting absorbance at $1378 \mathrm{~cm}^{-1}$ is then used to calculate the number of methyl groups per 100 carbon atoms. The conversion of absorbance to methyls per 100 carbon atoms is computed from the absorbance at $1378 \mathrm{~cm}^{-1}$ due to methyl groups in $n$-hexadecane (cetane).

\section{Experimental Procedure}

Films were prepared from SRM 1475 pellets taken from each container and blended as described in the first paper of this series [6]. In accordance with the ASTM procedure, several films ranging in thickness from 0.01 to $0.04 \mathrm{~cm}$ were molded in a hydraulic press heated to $168{ }^{\circ} \mathrm{C}$. The molten polyethylene in the mold assembly was then quenched in an ice-water mixture. Films prepared in this manner are designated reference films. Four films about $0.05 \mathrm{~cm}$ thick were also molded from the SRM pellets by the above procedure except that these films were annealed so as to produce an increase in density of at least $0.02 \mathrm{~g} / \mathrm{cm}^{3}$ greater than the density of the quenched reference films. The annealed films will later be referred to as

\footnotetext{
${ }^{2}$ Available from American Society for Testing and Materials, 1916 Race Street, Philadelphia, Pennsylvania 19103.
} 
samples I through IV. Difference spectra of each sample (high crystalline content) were obtained with three or four of the thinner reference films (low crystalline content). The spectra were recorded in the region from about 1430 to $1250 \mathrm{~cm}^{-1}$ using the prism optics of a double-beam, infrared spectrophotometer, (Perkin-Elmer Model 221). ${ }^{3}$ From these spectra, which range from partial to over-compensation of the absorption due to amorphous methylene groups, the absorbance at the methyl frequency, $A_{1378}$, and that at the methylene frequency, $A_{1304}$, were determined as directed by the ASTM method.

\section{Results and Discussion}

Effective absorptivities, $\alpha$, at 1378 and $1304 \mathrm{~cm}^{-1}$ were calculated from the difference spectrum of each sample-reference pair by the relation $\alpha=A /\left(d_{s} t_{s}\right.$ $-d_{r} t_{r}$ ), where $A$ is absorbance and $d_{s}, t_{s}, d_{r}$ and $t_{r}$ are sample density, sample thickness, reference density, and reference thickness, respectively. The resulting pairs, $\left(\alpha_{1304}, \alpha_{1378}\right)$, were plotted as shown in figure 1 , together with the straight line determined by a linear least squares fit. The value of $\alpha_{1378}$, on the least squares line, corresponding to $\alpha_{1304}=0$ is the absorbtivity in $\mathrm{cm}^{2} / \mathrm{g}$ at $1378 \mathrm{~cm}^{-1}$ at which the methylene absorptivity at $1304 \mathrm{~cm}^{-1}$ is completely compensated. This quantity is designated $K^{\prime}$ in table 1 . The number of methyl groups per 100 carbon atoms is also shown in table 1. The methyl concentration is computed from the product of $K^{\prime}$ and a calibration factor derived from measurements on cetane, as described in the ASTM procedure.

High density (linear) polyethylene chains may be presumed to be terminated with methyl groups as demonstrated by Willbourn [4] in a study of chain branching in polyethylene. If we assume two methyl groups per chain, then our estimate of 0.15 methyl groups per 100 carbon atoms leads to an estimate of 18,700 for the number-average molecular weight, $M_{n}$. This value closely approximates the $M_{n}(18,310)$ from the gel permeation chromatography measurement as shown in Paper $\mathrm{X}$ of this series [7]. This correlation and the density [8] suggests that the polymer, SRM 1475 , is essentially free of branching.

${ }^{3}$ Certain commercial equipment, instruments, or materials are identified in this paper in order to adequately specify the experimental procedure. In no case does such identifi cation imply recommendation or endorsement by the National Bureau of Standards, nor does it imply that the material or equipment identified is necessarily the best available for the purpose.

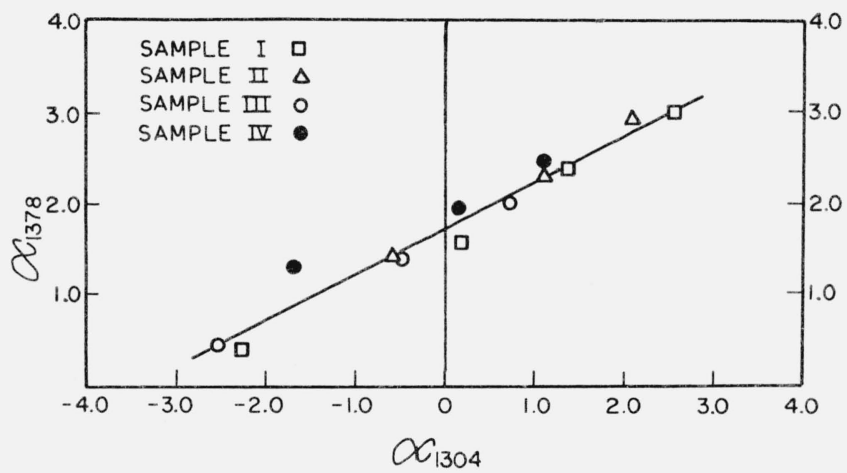

Figure 1. Ordinate: Absorptivity, $\alpha_{1378}, \mathrm{~cm}^{2} / g$; Abscissa: Absorptivity, $\alpha_{1304}, \mathrm{~cm}^{2} / g$.

The line shown in this graph represents a least squares fit of the absorptivity at $1378 \mathrm{~cm}^{-1}$ resulting from methyl groups against the absorptivity at $1304 \mathrm{~cm}^{-1}$ arising from amorphous methylene groups. The slope is equal to $0.51 \pm 0.03$ with the intercept at $1.74 \pm 0.05$ where the symbol \pm stands for the standard deviation in these quantities inferred from the linear least-squares fit. The intercept represents the methyl absorption at perfect compensation
lone for the methylene groups.

TABLE 1. Results of methyl group determination from compensated spectra

\begin{tabular}{c|c|c}
\hline \hline $\begin{array}{c}\text { Absorptivity } \\
K^{\prime}, \mathrm{cm}^{2} / \mathrm{g}\end{array}$ & $\begin{array}{c}\text { Sample standard } \\
\text { deviation of } K^{\prime}\end{array}$ & $\begin{array}{c}\mathrm{CH}_{3} \text { groups per } \\
100 \mathrm{C} \text { atoms }\end{array}$ \\
\hline 1.74 & 0.053 & 0.15 \\
\hline
\end{tabular}

$K^{\prime}$ in $\mathrm{cm}^{2} / \mathrm{g}$ is the reduced absorptivity of methyl groups, at complete compensations for the absorption due to methylene groups determined for SRM 1475.

\section{References}

[1] Cross, L. H., Richard, R. B., and Willis, H. A., Discussions Faraday Soc., No. 9, 235 (1950).

[2] Nielson, J. R. and Holland, R. F., J. Mol. Spectr. 4, 488 (1960).

[3] Okada, T. and Mandelkern, L., J. Polymer Sci. A-2 5,239 (1967).

[4] Willbourn, A. H., J. Polymer Sci. 34, 569 (1959).

[5] Krimm, S., Liang, C. Y., and Sutherland, G. B. B. M., J. Chem. Phys. 25, 549 (1956).

[6] Hoeve, C. A. J., Wagner, H. L., and Verdier, P. H., J. Res. Nat. Bur. Stand. (U.S.), 76A (Phys. and Chem.), No. 2, 137-140 (Mar-Apr. 1972). Paper I of this series.

[7] Ross, G. S. and Frolen, L. J., J. Res. Nat. Bur. Stand. (U.S.), 76A (Phys. and Chem.), No. 2, 163-170 (Mar.-Apr. 1972). Paper $\mathrm{X}$ of this series.

[8] Brown, J. E., J. Res. Nat. Bur. Stand. (U.S.), 76A (Phys. and Chem.), No. 2, 143-144 (Mar--Apr. 1972). Paper III of this series.

(Paper 76A-707) 